Published in "Trends in Biochemical Sciences doi: 10.1016/j.tibs.2017.05.005, "

which should be cited to refer to this work.

\title{
A Puzzle of Life: Crafting Ribosomal Subunits
}

Dieter Kressler, ${ }^{1, \star}$ Ed Hurt, ${ }^{2, \star}$ and Jochen Baßler ${ }^{2, \star}$

The biogenesis of eukaryotic ribosomes is a complicated process during which the transcription, modification, folding, and processing of the rRNA is coupled with the ordered assembly of $\sim 80$ ribosomal proteins ( $r$-proteins). Ribosome synthesis is catalyzed and coordinated by more than 200 biogenesis factors as the preribosomal subunits acquire maturity on their path from the nucleolus to the cytoplasm. Several biogenesis factors also interconnect the progression of ribosome assembly with quality control of important domains, ensuring that only functional subunits engage in translation. With the recent visualization of several assembly intermediates by cryoelectron microscopy (cryo-EM), a structural view of ribosome assembly begins to emerge. In this review we integrate these first structural insights into an updated overview of the consecutive ribosome assembly steps.

\section{Synopsis of Eukaryotic Ribosome Assembly}

Ribosomes are the molecular machines that translate the genetic information from the intermediary mRNA templates into proteins [1]. Eukaryotic $80 S$ ribosomes comprise two unequal subunits that contain four different rRNAs and around 80 r-proteins (Figure 1). The small $40 S$ subunit (SSU) comprises the 18S rRNA and 33 r-proteins (referred to as RPS or S). The large $60 S$ subunit (LSU) comprises the 25S/28S, 5.8S, and 5 S rRNA and, in most eukaryotic species, 47 r-proteins (RPL or L); a notable exception is budding yeasts, which lack eL28 $[1-4]$.

The act of building a ribosome begins in the nucleolus, where the ribosomal DNA (rDNA) is transcribed into a long pre-rRNA precursor (35S pre-rRNA in yeast) and involves the ordered assembly of the r-proteins with the pre-rRNA, which is concomitantly processed into the mature rRNA species [5-8] (Figure 1 and Box 1). These assembly and processing events are tightly coupled and occur within preribosomal particles (see Glossary) that travel, as maturation progresses, from the nucleus across nuclear pore complexes (NPCs) to the cytoplasm, where they are ultimately converted into translation-competent ribosomal subunits [5,9-13] (Figure 2, Key Figure). Given the gargantuan complexity of this process, it is unsurprising that the assembly of eukaryotic ribosomes strictly requires the assistance of a plethora (>200) of mostly essential ribosome biogenesis factors, which are also called trans-acting or assembly factors $[5,14,15]$.

Our current knowledge has been mainly obtained by studying ribosome biogenesis in the yeast Saccharomyces cerevisiae. Research conducted in the 1970s defined the r-protein composition of ribosomes, revealed the major pre-rRNA processing intermediates, and uncovered the existence of the 90S, 43S, and 66S preribosomal particles. The following 25 years witnessed the identification of numerous biogenesis factors and attributed functional roles
Trends

Cryoelectron microscopy analyses of the $90 \mathrm{~S}$ preribosome show that the biogenesis factors create a casting mold that encloses the nascent pre405 subunit.

Structures of nuclear pre-60S particles reveal that accommodation of the $5 \mathrm{~S}$ ribonucleoprotein into its final position involves major structural rearrangements of the central protuberance. Moreover, within the 'foot' region the internal transcribed spacer (ITS) 2 rRNA is bound by several biogenesis factors that coordinate ITS2 processing.

An increasing number of biogenesis factors can be viewed as checkpoint factors sensing the successful completion of assembly events before licensing preribosomes for the subsequent maturation steps.

Supplying all ribosomal building blocks in stoichiometric amounts is a highly regulated process. Several dedicated chaperones contribute to the production of assembly-competent ribosomal proteins (r-proteins). Excess r-proteins are degraded via the excess $r$-protein quality control pathway.

${ }^{1}$ Unit of Biochemistry, Department of Biology, University of Fribourg, Chemin du Musée 10, CH-1700 Fribourg, Switzerland

${ }^{2}$ Biochemistry Center Heidelberg (BZH), University of Heidelberg, Im Neuenheimer Feld 328, D-69120 Heidelberg, Germany

${ }^{*}$ Correspondence:

dieter.kressler@unifr.ch (D. Kressler), ed.hurt@bzh.uni-heidelberg.de (E. Hurt),

jochen.bassler@bzh.uni-heidelberg.de (J. Baßler). 
35S pre-rRNA
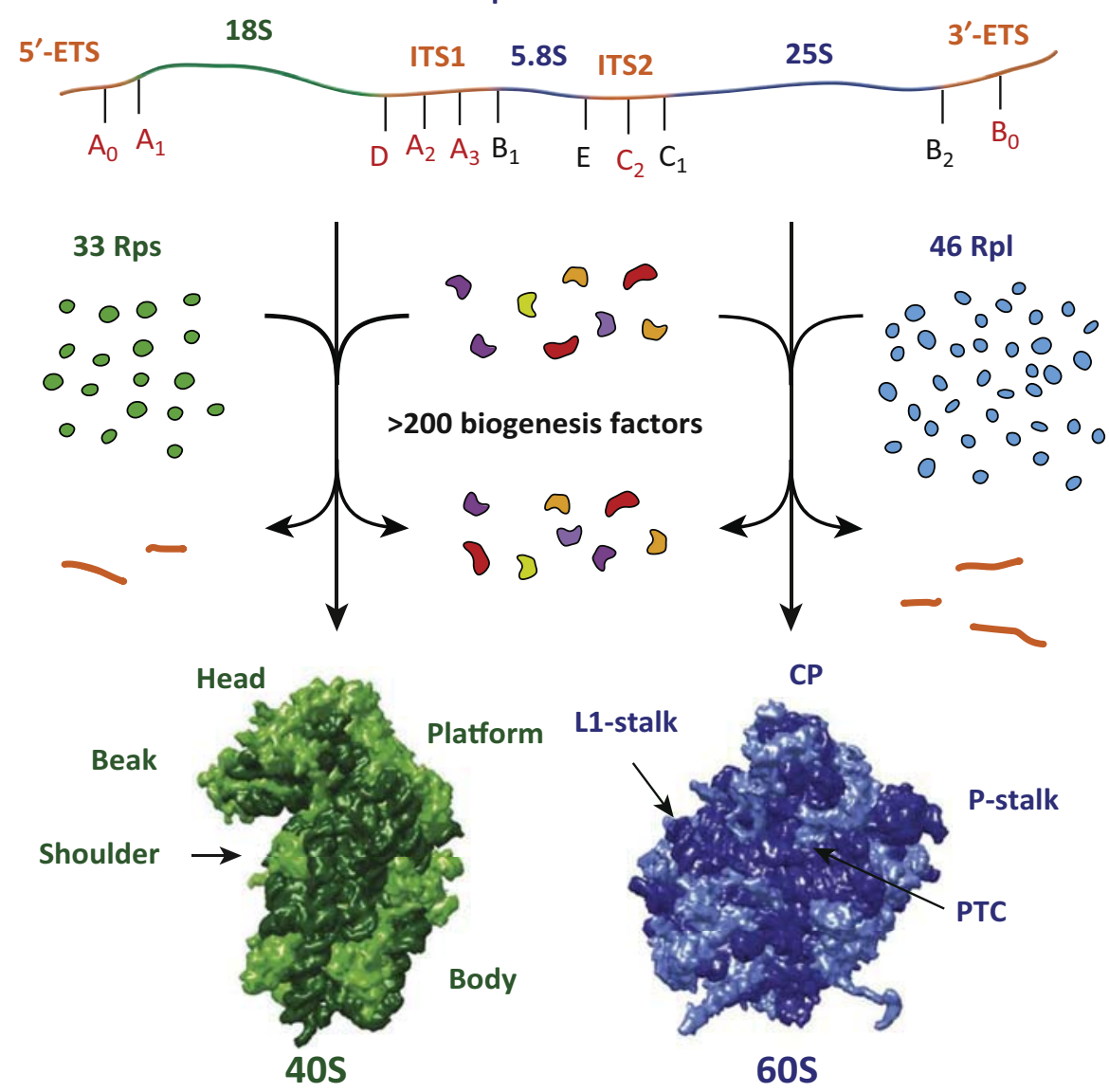

Trends in Biochemical Sciences

Figure 1. Schematic Representation of Ribosome Biogenesis in Saccharomyces cerevisiae. Ribosome biogenesis comprises the assembly of rRNA (top) and ribosomal proteins (r-proteins), which is coordinated and catalyzed by more than 200 biogenesis factors. $r$-Proteins of the $40 S$ (light green) and $60 S$ (light blue) subunits are synthesized from mRNA transcripts produced by RNA polymerase II. The 35S pre-rRNA and the 5S rRNA (dark blue) are synthesized by RNA polymerase I and RNA polymerase III, respectively. During ribosome biogenesis the external transcribed spacer (ETS) and internal transcribed spacer (ITS) (orange) are removed from the 35S pre-rRNA to obtain the mature 18S (dark green), 25S, and 5.8S (dark blue) rRNAs. These rRNA maturation events include a series of endo- and exonucleolytic processing reactions whose cleavage and trimming sites are indicated by red and black letters, respectively. The mature ribosome comprises two asymmetric subunits: the small $40 \mathrm{~S}$ subunit contains 33 r-proteins (Rps; light green) and the 18S rRNA (dark green) while the large 60S subunit comprises 46 r-proteins (Rpl; light blue) and the 5.8S, 25S, and 5S rRNAs (dark blue). Structural hallmarks of the two mature ribosomal subunits are indicated, including the central protuberance (CP), which comprises the 5S rRNA and its associated r-proteins. Formation of new peptide bonds occurs in the peptidyl transferase center (PTC) of the 60S subunit. Surface representations of the mature $40 S$ and $60 S$ subunits from $S$. cerevisiae, shown in the interface view, were generated with Chimera using PDB 4V88 [113].

to these in the assembly and export of preribosomal subunits as well as the modification and processing of pre-rRNAs [16-18]. At the beginning of this millennium, the implementation of powerful in vivo affinity-purification approaches permitted the isolation and characterization of preribosomal particles $[19,20]$. Subsequently, the purification of additional preribosomal particles provided a detailed spatiotemporal picture of the preribosomal intermediates and their composition [5,9,14] (Figure 2). A few of these preribosomal particles were visualized at relatively low resolution by EM [21-24]. Recent progress in cryo-EM has enabled a quantum jump by permitting the visualization of preribosomal particles at atomic or near-atomic resolution [25-33]. These studies not only provided a detailed view of the overall architecture of

\section{Glossary}

Biogenesis factors: the efficient and accurate assembly of ribosomal subunits is promoted by more than 200 proteins that are transiently associated with preribosomal particles; these proteins are collectively referred to as biogenesis factors, or alternatively as transacting or assembly factors.

Chaetomium thermophilum: a thermophilic filamentous ascomycete whose optimal growth temperature is around $50-55^{\circ} \mathrm{C}$. Proteins and macromolecular complexes of this eukaryotic organism often exhibit improved properties that can be exploited for biochemical and structural studies.

Decoding center: functional center of the SSU where decoding of mRNA occurs by selection of the correct base pairing between the codon and the anticodon of the cognate aminoacyl-tRNA.

External and internal transcribed spacer (ETS/ITS): the 35S prerRNA is flanked by $5^{\prime}$-ETS and $3^{\prime}$ ETS sequences and contains the internal ITS1 and ITS2, which separate the mature rRNA sequences; See also 'Pre-rRNA processing' and Figure 1.

Peptidyl transferase center (PTC): the active site of the LSU where the formation of a new peptide bond is catalyzed.

Preribosomal particle: maturation of ribosomal subunits occurs within preribosomal particles (also termed preribosomes) comprising (pre-) rRNA, r-proteins, and biogenesis factors (Figure 2).

Pre-rRNA processing: series of reactions that generate the mature rRNA species (18S, 5.8S, 25S, and $5 S$ rRNA) from the large $35 \mathrm{~S}$ prerRNA and the pre-5S rRNA. During processing of the $35 \mathrm{~S}$ pre-rRNA, the ETS and ITS are removed by the action of endo- and exonucleases (Figure 1).

P stalk: characteristic feature of 605 subunits comprising a pentameric module of acidic r-proteins; contributes to the binding of translational GTPases.

Ribosomopathies: a group of inherited diseases caused by mutations in r-proteins or biogenesis factors that affect ribosome biogenesis or translation efficiency. U3 small nucleolar ribonucleoprotein particle 
Box 1. Basic Steps of Eukaryotic Ribosome Assembly

- Transcription of rDNA into pre-RNA and modification of the nascent pre-rRNA by snoRNPs (ribose methylation and formation of pseudouridines) in the nucleolus.

- Cytoplasmic synthesis of $r$-proteins and biogenesis factors and, for the majority, subsequent transport to the nucle(ol)us.

- Spatiotemporal association of biogenesis factors and r-proteins with the pre-rRNA results in the formation of preribosomal particles.

- Processing of the pre-rRNA (endo- and exonucleolytic removal of ETS and ITS sequences) separates the maturation pathways of the two subunits and is coordinated with the progression of ribosome assembly.

- Export of preribosomes from the nucleus across the nuclear pore complex to the cytoplasm.

- Final maturation of both preribosomal subunits occurs in the cytoplasm and includes the incorporation of the last $r$ proteins as well as the release and recycling of biogenesis factors.

- Quality control steps, involving several nuclear and cytoplasmic checkpoints, ensure that only fully functional ribosomal subunits can participate in the translation process.

preribosomal particles but also displayed numerous biogenesis factors in the structural context of preribosomal particles. Here we outline the current view of the ribosome assembly process in yeast in the context of the recent functional and structural advances.

\section{Providing a Balanced Supply of Ribosomal Components}

To guarantee the efficient assembly of ribosomes, cells are faced with the enormous logistic challenge of producing equal amounts of the four rRNAs and the 79 r-proteins (Figure 1). The transcription of rDNA and r-protein-encoding genes (RPGs) is connected to growth conditions, which are relayed via the TORC1 kinase [34], but a balanced production is complicated because the transcription of these components includes all three RNA polymerases [5,34]. While common promoter elements ensure the harmonized transcription of RPGs [34], a recent study revealed how a negative feedback loop links early assembly events with RPG transcription. A shortage of nascent pre-rRNAs leads to an increased free pool of the 905 biogenesis factor Utp22, which then sequesters the transcriptional activator Ifh1 from RPG promoters [35]. However, both the quantitative and qualitative production of $r$-proteins clearly depends also on several post-transcriptional events, including the stability and splicing efficiency of RPG mRNAs as well as the folding and intrinsic stability of r-proteins and, for most of these, their transport into the nucleus $[6,36]$. Therefore, the constant production of $r$-proteins in somewhat greater quantity than required seems advantageous for avoiding a shortage of these building blocks during ribosome assembly.

Prominent features of a large number of $r$-proteins are unusual overall folds and a high percentage of basic amino acids, which often cluster in long extensions and internal loops that are, in many instances, involved in rRNA binding [1]. Owing to these properties, $r$-proteins are especially prone to aggregation and general ribosome-associated chaperone systems contribute to their soluble expression $[37,38]$. In addition, a heterogeneous class of proteins, collectively referred to as dedicated chaperones, specifically protects individual r-proteins and safely guides them to their assembly site on preribosomal particles [37] (Figure 2A). So far, dedicated chaperones have been shown to associate with eight of the 79 yeast $r$-proteins $[37,39]$. Notably, the majority of these already capture their $r$-protein clients during translation $[40,41]$.

The ubiquitin-proteasome system rapidly degrades unincorporated r-proteins, thereby antagonizing their aggregation bias [42,43]. To avoid targeting preribosomes for degradation, this nuclear quality control system, termed excess r-protein quality control (ERISQ), specifically ubiquitinates those lysine residues of $r$-proteins that are no longer accessible after their assembly into preribosomes [43]. Kinetic competition for r-proteins between ERISQ and the preribosome might constitute a mechanism to selectively eliminate excessive r-proteins [43]. Furthermore, association with dedicated chaperones and/or importins may prevent
(snoRNP): the U3 snoRNP contains the C/D-box snoRNA U3, all core C/ D-box snoRNP proteins, and the U3specific protein Rrp9. The U3 snoRNP is a structural component of the 905 preribosome and is essential for the early pre-rRNA processing reactions.

UTP-A/UTP-B/UTP-C: the UTP (U three protein) complexes are three independent assembly modules whose components were originally identified as factors associated with the U3 snoRNA. The letters A, B, and $C$ denote their order of assembly into the $90 \mathrm{~S}$ preribosome. 

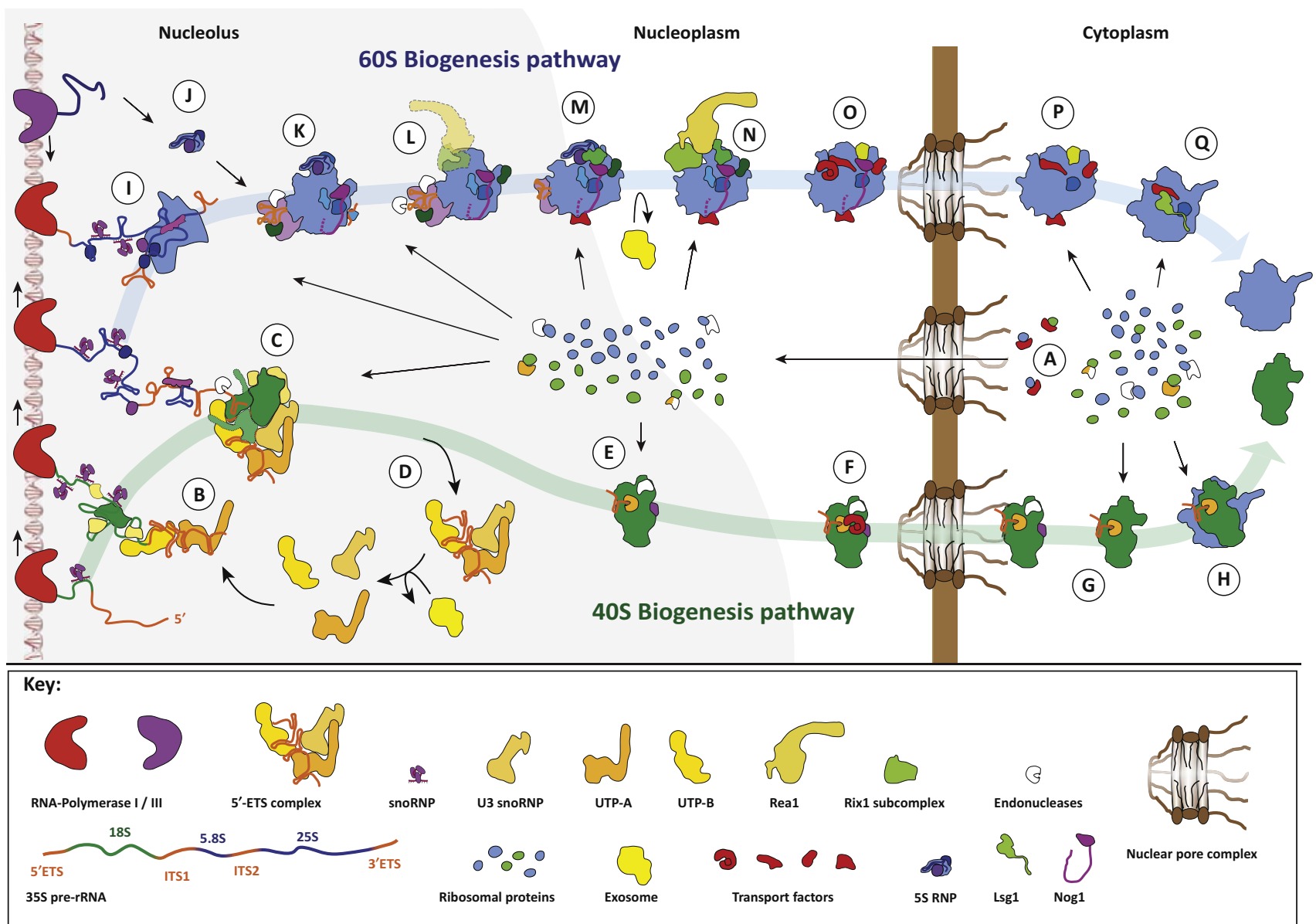

Trends in Biochemical Sciences

Figure 2. The majority of ribosomal proteins (r-proteins) (A) need to be transported from the cytoplasm to their assembly sites within the nucleus. The assembly pathways leading to the formation of mature $40 \mathrm{~S}$ [green; lower pathway from left to right $(\mathrm{B}-\mathrm{H})$ ] and $60 \mathrm{~S}$ subunits [blue; upper pathway from left to right $(\mathrm{I}-\mathrm{Q})$ ] originate from a common pre-rRNA (35S pre-rRNA) that is transcribed by RNA polymerase I. Distinct assembly modules, biogenesis factors, and r-proteins associate cotranscriptionally with the nascent pre-rRNA (B) to form a 90 S preribosome (C). Dismantling of the $90 \mathrm{~S}$ preribosome and pre-rRNA cleavages liberate the $5^{\prime}$-external transcribed spacer (ETS) complex, whose components are recycled for further assembly rounds (D), and the first pre-40S particle (E). Export factors mediate the transport of pre-40S ribosomes to the cytoplasm (F) where they undergo further maturation steps, including beak formation (G). Final $40 \mathrm{~S}$ maturation occurs within $80 \mathrm{~S}$ like ribosomes and couples pre-rRNA cleavage with a quality control step $(\mathrm{H})$. The first pre-60S particle, whose components begin to associate with the nascent prerRNA, is likely to be formed after internal transcribed spacer 1 (ITS1) cleavage and termination of transcription (I). Then, the preformed $5 S$ ribonucleoprotein particle (RNP) (J) associates with early pre-60S particles (K), which already contain the ITS2-associated biogenesis factors. Subsequently, ITS2 processing is initiated and the first Rea1-dependent remodeling step occurs (L). The Nog2-purified pre-60S particle exhibits the prominent 'foot' structure and the 5S RNP in its pre-mature position (M). The second Rea1-dependent remodeling step coincides with rotation of the 5S RNP into its mature position and occurs in concert with the release of the GTPase Nog2 $(\mathrm{N})$, which is a prerequisite for recruitment of the export adaptor $\mathrm{Nmd} 3(\mathrm{O})$ and translocation through the nuclear pore complex. In the cytoplasm final maturation and quality control steps yield mature 60S subunits (P,Q). See main text for detailed descriptions of the highlighted assembly steps. 
r-proteins from being ubiquitinated [37,43], thereby enabling their storage until they are incorporated into preribosomes.

$r$-Proteins are important not only for the structure and function of the ribosome but also for its assembly [6]. Whereas severe mutations in r-proteins will produce non-functional ribosomes, milder mutations may cause biogenesis and/or translation defects. In humans, reduced translation efficiency or accuracy may lead to ribosomopathies, whose clinical characteristics often include anemia or developmental defects [44-47]. Moreover, defects in ribosome assembly increase the levels of free r-proteins, acting as a signal for ribosome biogenesis stress. Accordingly, in multicellular organisms, elevated levels of uL 5 and uL18 are functionally linked to the E3 ubiquitin ligase MDM2 leading to accumulation of its substrate p53, thereby blocking cell division $[48,49]$. Taking these findings together, the levels of free r-proteins are well regulated and monitored by various mechanisms, such as transcriptional regulation, association with binding partners, and degradation, to embed ribosome assembly into other cellular pathways that exert growth control.

\section{Assembly of the SSU}

Building the 905 Preribosome

Before folding, the nascent pre-rRNA is extensively modified by 2'-O-ribose methylation and isomerization of uridines to pseudouridines (Figure 2B). The modified sites, which cluster in functional regions of the ribosome, are identified by complementary base pairing between guide small nucleolar RNAs (snoRNAs) and rRNA [50]. These snoRNAs fall into two classes based on their integration into C/D-box or H/ACA-box small nucleolar ribonucleoprotein particles (snoRNPs), which have methyltransferase or pseudouridine synthase activity, respectively [50]. In yeast, 42 C/D-box snoRNAs and 28 H/ACA-box snoRNAs target around 100 different nucleotides for modification [51]. To allow accurate folding of pre-rRNA and its assembly with $r$-proteins, snoRNAs must dissociate from the rRNA. These processes involve DExD/H-box RNA helicases, which can generally be viewed as RNA chaperones or RNP remodelers $[50,52,53]$.

Already during rDNA transcription, the first biogenesis factors and $r$-proteins associate with the nascent 35S pre-rRNA to form the $90 \mathrm{~S}$ preribosome/SSU processome $[5,9,54]$. Most of these biogenesis factors form well-defined complexes, including the U3 snoRNP and the UTP-A, UTP-B, and UTP-C modules, which assemble in a stepwise manner with the pre-rRNA $[5,55]$ (Figure 2B). Several recent studies have significantly advanced our understanding of these initial assembly events. Biochemical reconstitution and structural approaches revealed the architecture of the UTP-A and UTP-B modules [56-60]. Crosslinking analyses supported the idea that UTP-A is the first module to associate with the $5^{\prime}$-external transcribed spacer (ETS) region of the nascent 35 S pre-rRNA $[27,58]$ (Figures 1, 2B, and 3 ). Subsequently, the UTP-B complex binds downstream of UTP-A, but still within the $5^{\prime}$-ETS region. Thus, the UTP-A and -B modules might predominantly contribute to the structural nucleation of the evolving 905 preribosome (Figures 2B,C and 3). To obtain more insight into the timing of 905 assembly, two groups expressed various $3^{\prime}$ truncations of the pre-18S rRNA and analyzed the associated proteome, thereby revealing the gradual binding of distinct clusters of biogenesis factors $[61,62]$. Notably, relatively few biogenesis factors dissociate or show reduced association as formation of the $90 \mathrm{~S}$ preribosome reaches completion [61]. If one hypothesizes a strict hierarchical order, these isolated complexes should represent the early, intermediate, and late stages of $90 \mathrm{~S}$ ribosome assembly.

\section{Architecture of the 90S Preribosome}

The 905 preribosome appears to represent the first stable preribosomal particle (Figures 2C and 3). Accordingly, in vivo purification of UTP-A or -B components permits the isolation of 

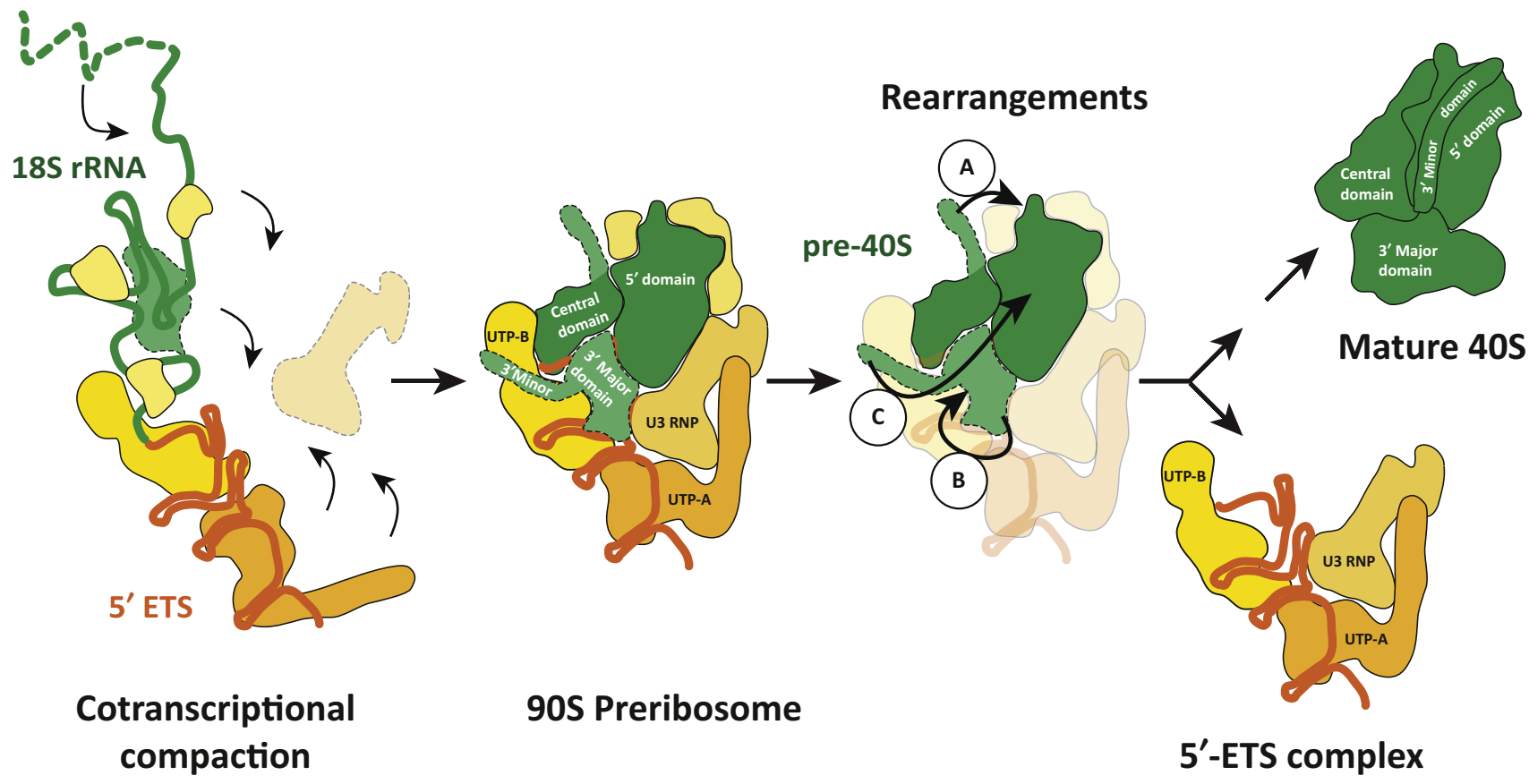

Trends in Biochemical Sciences

Figure 3. Model of 90S Formation and Its Conversion into Pre-40S Ribosomes. The UTP-A, UTP-B, and U3 small nucleolar ribonucleoprotein particle (snoRNP) modules associate cotranscriptionally with the 35S pre-rRNA. Presumably, subsequent compaction leads to formation of the $90 \mathrm{~S}$ preribosome, where the biogenesis factors (yellow, orange) form a casting mold for the nascent $18 \mathrm{~S}$ rRNA (green). While the overall fold of the $5^{\prime}$ domain of the $18 \mathrm{~S} r \mathrm{RNA}$ is already similar to its final conformation, several major structural rearrangements within the central, $3^{\prime}$ major, and $3^{\prime}$ minor domains are required to transform the pre-40S moiety of the $90 S$ preribosome into the mature 40S subunit. (A) The eukaryote-specific expansion segment ES6 needs to be completely folded and moved towards the $5^{\prime}$ domain. (B) The $3^{\prime}$ major domain requires further stabilization and folding to form the characteristic head structure, which still has to be rearranged to acquire its final position. (C) The $3^{\prime}$ minor domain, mainly comprising the long helix $\mathrm{H} 44$, has to undergo a dramatic movement to be accommodated at its binding site on the $5^{\prime}$ domain. However, the timing and mechanisms of these rearrangements remain to be determined. Release of the $5^{\prime}$-external transcribed spacer (ETS) complex and endonucleolytic cleavage within internal transcribed spacer 1 (ITS1) (not depicted) liberate the first pre-40S particle, which is converted in further biogenesis steps into the mature $40 \mathrm{~S}$ subunit.

preribosomal particles comprising a 5'-ETS-containing pre-18S rRNA, about 20 SSU r-proteins, 65 biogenesis factors, and the U3 snoRNP [27,29]. A major recent breakthrough is the determination of cryo-EM structures of the $90 \mathrm{~S}$ preribosome derived from Chaetomium thermophilum and S. cerevisiae [27,29,31] (Figure 4A). All three structures unveil the features of the nascent $40 S$ moiety, revealing how most of the pre-rRNA, about 30 biogenesis factors, and 15 SSU r-proteins are arranged within this 5-MDa assembly intermediate $[27,29,31]$. The UTP-A and UTP-B modules are bound to the rRNA helices of the $5^{\prime}$ ETS and form, together with the U3 snoRNP and additional biogenesis factors, a stable building block of the $90 \mathrm{~S}$ preribosome. Strikingly, the biogenesis-factor ensemble forms a casting mold that engulfs the nascent pre-18S rRNA (Figures 3 and 4), thereby protecting it from degradation and enabling its accurate processing and controlled folding. The $5^{\prime}$ domain of the 18S rRNA exhibits significant similarity to its mature conformation and already contains the majority of its ultimately associated $r$-proteins. Most of the central domain is properly positioned relative to the $5^{\prime}$ domain and already harbors some r-proteins at their cognate binding sites. The $3^{\prime}$ major domain is completely buried in the center of the $90 \mathrm{~S}$ preribosome and its conformation is significantly distinct from that in the mature state, clearly indicating a hierarchical, $5^{\prime}$-to- $3^{\prime}$-oriented assembly process. The cryo-EM structures further revealed how the essential U3 snoRNA engages in base-pairing interactions with the $5^{\prime}$ ETS and the pre-18S rRNA (Figures 3 and 4). An interesting hypothesis is that the U3 snoRNP not only stimulates pre-rRNA folding and processing but also locks the 905 preribosome in an intermediate folding state. This may provide a necessary time 
(A)

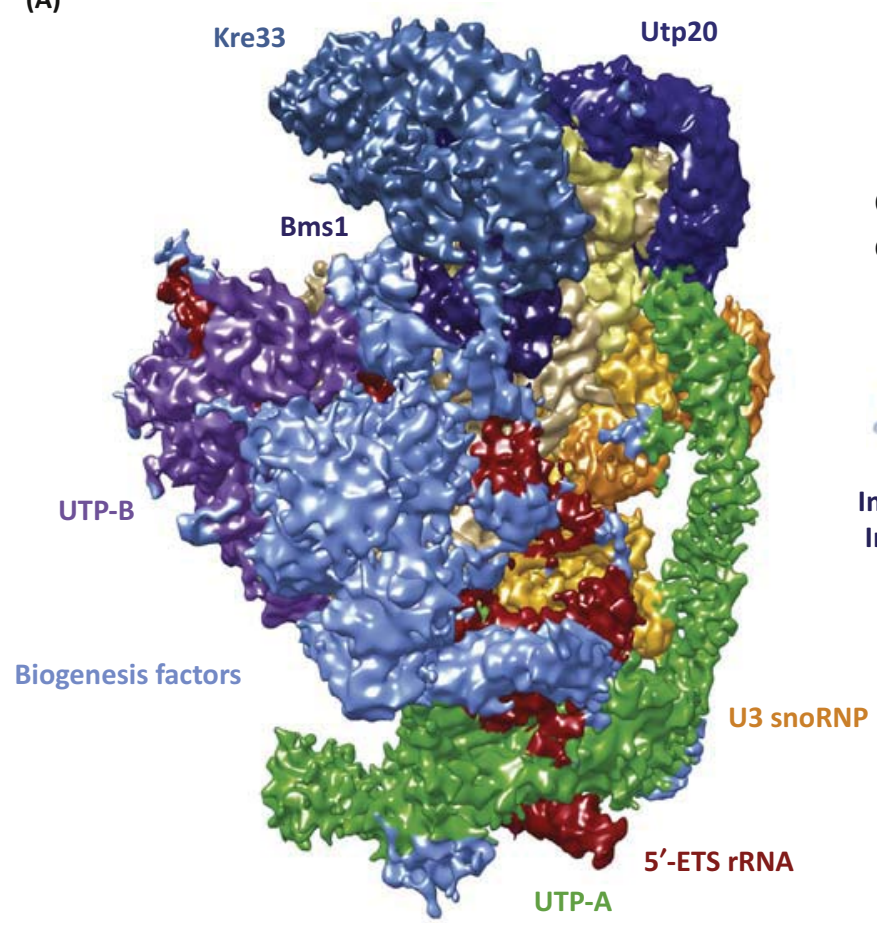

(B)

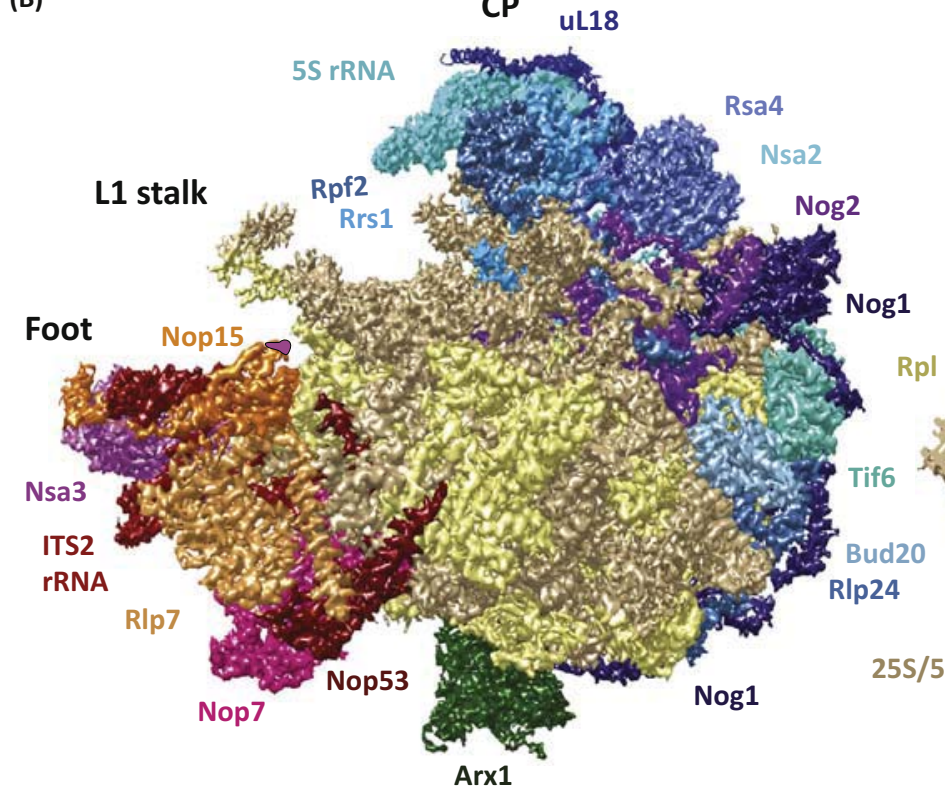

5' Domain

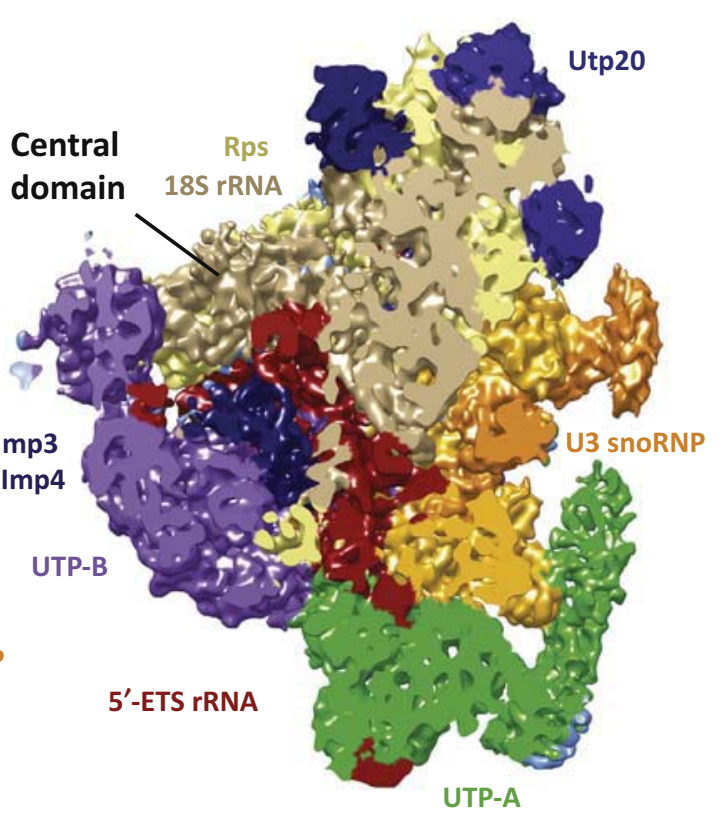

CP

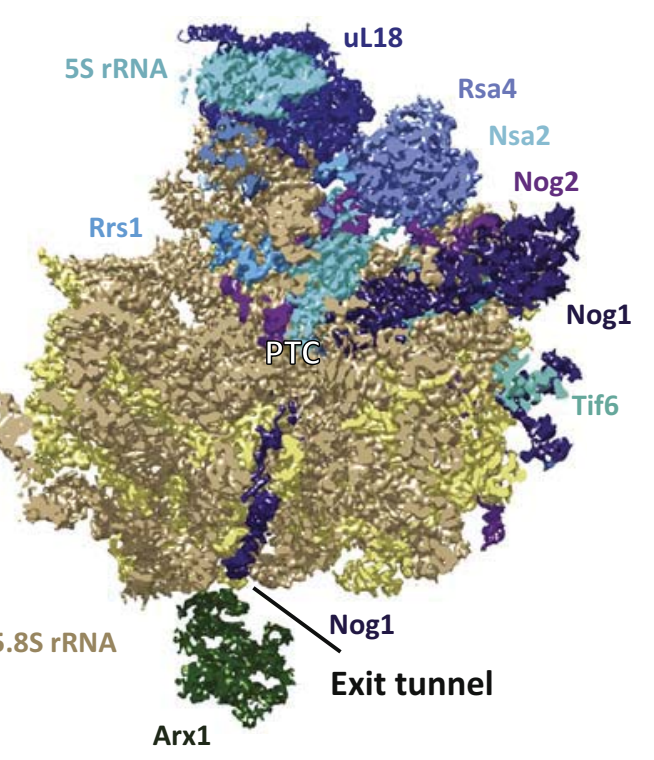

Trends in Biochemical Sciences

Figure 4. A Structural View of Selected Preribosomal Particles. (A) Cryoelectron microscopy (cryo-EM) structure of the 90S preribosome from Chaetomium thermophilum (EMD 8143) [27]. The indicated assembly modules and biogenesis factors are highlighted in different colors. The remaining biogenesis factors are colored in light blue. The $5^{\prime}$ external transcribed spacer (ETS) (red), the 18S rRNA (tan), and the ribosomal proteins ( $r$-proteins) (Rps; khaki) are also highlighted. A cross-section of the $90 \mathrm{~S}$ preribosome reveals the interior of the cage-like structure (right panel). The positions of the $5^{\prime}$ domain and the central domain of the $18 \mathrm{~S} r \mathrm{RNA}$ within the nascent pre-40S moiety are indicated. (B) Cryo-EM structure of the Nog2-purified pre-60S ribosome from Saccharomyces cerevisiae (EMD 6615) [25]. Characteristic features, including the 'foot', L1 stalk, and central protuberance (CP), are indicated. The prominent foot structure, comprising several biogenesis factors (Nop7, Nop15, Nop53, Nsa3, and RIp7) and the internal transcribed spacer 2 (ITS2) rRNA, is highlighted. In its pre-mature orientation, the 5S ribonucleoprotein particle (RNP) (5S rRNA and r-proteins uL18 and uL5) contacts the Rpf2-Rrs1 heterodimer. The 25S/5.8S rRNAs are colored in tan and the r-proteins in khaki. A cross-section of the pre-60S ribosome (right panel) shows the insertion of Nog1's C-terminal extension (dark violet) into the polypeptide exit tunnel and the interconnection of biogenesis factors (Nsa2, Nog1) in proximity to the peptidyl transferase center (PTC). 
window for biogenesis events to occur before the release of the $5^{\prime}$ ETS and the subsequent formation of the central pseudoknot structure that interconnects the four $18 \mathrm{~S}$ rRNA domains.

Dismantling of the 90S Preribosome Releases an Early Pre-40S Particle

Despite our detailed knowledge of the pre-rRNA processing pathway in yeast and human cells $[7,63]$, the enzymes that cleave the pre-rRNA at sites $A_{0}, A_{1}$, and $A_{2}$ have not yet been unambiguously assigned. While all three cleavages depend on the U3 snoRNP, processing at sites $A_{1}$ and $A_{2}$ appears to be coupled and occurs independently of $A_{0}$ cleavage [7]. Recent evidence strongly suggests that the $90 \mathrm{~S}$-associated Utp24 is the endonuclease responsible for cleavages at sites $A_{1}$ and $A_{2}$ [64]. This PIN-domain endonuclease is positioned in proximity to the $A_{1}$ cleavage site $[27,29,64]$ and cleaves a pre-rRNA substrate at site $A_{2}$ in vitro [64]. $A$ second 90S-associated endonuclease with in vitro $A_{2}$ cleavage activity is $\operatorname{Rcl1}[27,29,31,65]$. Unfortunately, the positions of the rRNA segments around sites $A_{0}$ and $A_{2}$ could not be assigned in the current $90 \mathrm{~S}$ structures $[27,29]$. To clarify and understand the direct contribution of Utp24 and Rcl1 to these early cleavages, it will be instrumental to generate improved $90 \mathrm{~S}$ cryo-EM structures. Such structures might also reveal how these early cleavages are coupled with progression to the first pre-40S particle. This transition requires the release of the $5^{\prime}$-ETS complex, which mainly comprises $5^{\prime}$-ETS rRNA, UTP-A and UTP-B modules, and U3 snoRNP [27] (Figures 2D and 3). Exosome-mediated degradation of the $5^{\prime}$-ETS rRNA may initiate the disassembly of the $5^{\prime}$-ETS complex, whose recycled components can then participate in a new round of $90 S$ assembly $[27,66]$. Dismantling of the 905 preribosome is likely to depend on the RNA helicase Dhr1, since pre-40S intermediates trapped by catalytically inactive Dhr1 still contain the U3 snoRNP [67]. Moreover, Dhr1, stimulated by its recruiting cofactor Utp14, can unwind U3 rRNA duplexes in vitro $[67,68]$. However, other helicases associated with the $90 \mathrm{~S}$ preribosome might also be required for the dismantling process that releases the early pre-40S particle. Clearly, further studies will be required to obtain a complete picture of these early biogenesis events.

\section{Final Maturation of Pre-40S Particles}

Cleavage of the pre-rRNA at site $A_{2}$ results in the separation of the biogenesis pathways of the SSU and LSU (Figure 2C), which will only meet again in the cytoplasm. The next biochemically characterized pre-40S intermediates following the 905 preribosome contain the $20 S$ pre-rRNA, which corresponds to an $18 \mathrm{~S}$ rRNA extended to site $\mathrm{A}_{2}$, and around ten biogenesis factors [69]. Notably, most of these biogenesis factors are exclusively associated with pre-40S particles; therefore, the transition from the $90 \mathrm{~S}$ to the first $40 \mathrm{~S}$ preribosome is hallmarked by an enormous exchange of biogenesis factors [69]. Moreover, several r-proteins, including the early binders of the $3^{\prime}$ major domain, need to be recruited at this stage. However, since no transition intermediates have been purified so far, the 90S-to-pre-40S conversion appears either to be a kinetically fast process or to involve unstable interjacent particles (Figure 2E).

Rapidly after their formation, pre-40S particles are exported through the NPC to the cytoplasm (Figure 2F). Due to the large size of the pre-40S ribosome, it is predicted that multiple export factors are required to facilitate its rapid translocation [70]. Pre-40S export depends on the GTPase Gsp1/Ran and Crm1/Xpo1, the general exportin for substrates harboring nuclear export signal (NES) sequences [5,10,71,72]. At least three biogenesis factors (Dim2, Ltv1, and Rio2) contain predicted or functional NESs that are, possibly due to their redundancy, individually not required for pre-40S export $[5,10]$. Additionally, several other factors have been implicated in the export of pre-40S subunits [10], but their direct role in this process remains vague.

In agreement with a hierarchical assembly pathway, the cytoplasmic maturation events mainly concern the $3^{\prime}$ major and $3^{\prime}$ minor domains. Moreover, the release of biogenesis factors, which 
occupy surfaces that later engage in interactions with translation factors $[73,74]$, must be coordinated with the accommodation of the last r-proteins and the cleavage of the 20S prerRNA [6]. First, formation of the characteristic beak structure involves the stable incorporation of uS3, which is triggered by the phosphorylation-dependent release of Ltv1 $[22,75,76]$ (Figure 2G). The Rio2 ATPase is strategically positioned between the body and the maturing head in proximity to the decoding center and subsequently may act as a self-releasing checkpoint factor $[73,77]$. Recruitment of the ATPase Rio1, presumably requiring the prior dissociation of Tsr1 [78], yields late pre-40S ribosomes that are competent to join 60 S subunits [79-81]. Within these 80S-like ribosomes, Rio1 and the GTPase elF5B stimulate the Nob1-catalyzed cleavage at site D of the 20S pre-rRNA into mature 18S rRNA $[74,80,82-84]$ (Figures 1 and $2 H$ ). Coupling pre-40S maturation to such a quality control step ensures that only properly assembled 40S subunits enter the pool of translating 805 ribosomes.

Taken together, structural investigations of the 90S preribosome have unraveled its detailed architecture; however, many functional and enzymatic aspects remain to be explored. Subsequent pre-rRNA processing and dismantling releases the first pre-40S particle, which is rapidly exported to the cytoplasm where final maturation and functional proofreading occur.

\section{Assembly of the LSU}

Nuclear 60S Biogenesis Events

Cleavage at site $A_{2}$ within internal transcribed spacer 1 (ITS1) liberates the SSU moiety from the pre-rRNA transcript and sets the stage for the assembly of the LSU (Figure 2C). Contrary to the relatively simple $40 S$ assembly route, which proceeds along the sequential, $5^{\prime}$-to- $3^{\prime}$ oriented folding of its four distinct 3D domains, 60S assembly appears more complex as its six secondary-structure rRNA domains are elaborately intertwined in the mature 60S ribosome [2,4]. Moreover, evidence gathered over the past 15 years has revealed that LSU assembly occurs within several distinct and successive pre-60S intermediates of partially overlapping composition (Figure 2). The initial steps of LSU assembly remain poorly characterized, but it appears that the formation of the first pre-60S particle depends on termination of transcription and the association of early-binding LSU r-proteins that connect the $5^{\prime}$ and $3^{\prime}$ regions of the $27 \mathrm{~S}$ pre-rRNA $[6,11]$ (Figure 2I). Evidence suggests that rRNA modification may not exclusively occur cotranscriptionally since the presumably earliest pre-60S precursor, isolated via the Npa1 bait protein, still contains C/D- and H/ACA-box snoRNPs [85]. Moreover, the presence of eight RNA helicases in this Npa1-defined particle, which mainly comprises the $27 \mathrm{SA}_{2}$ pre-rRNA, indicates that extensive rRNA folding is occurring.

The next nucleolar pre-60S particle, the Ssf1-defined particle, exhibits a complex composition: it contains a $27 \mathrm{~S}$ pre-rRNA intermediate, more than 30 biogenesis factors, and already around half of the LSU r-proteins [86-88]. The following pre-60S ribosomes (Erb1- or Nsa1associated particles) mainly comprise the $27 \mathrm{SB}$ pre-rRNAs and have already lost some early biogenesis factors $[86,89]$ (Figure $2 \mathrm{~K}$ ). This loss might be due to the trimming of the $27 \mathrm{~S}$ prerRNA at its $5^{\prime}$ and $3^{\prime}$ termini $\left(A_{3} \rightarrow B_{1}\right.$ and $B_{0} \rightarrow B_{2}$; Figure 1$)$. Moreover, the biogenesis factors that associate around the ITS2 region, forming the characteristic 'foot' structure (see below), have already been recruited at this stage [86]. A specific feature of LSU assembly is the incorporation of a preformed 5S RNP (Figure 2J) comprising the 5S RNA and the rproteins $\mathrm{UL} 5$ and uL18. Formation of the $5 \mathrm{~S}$ RNP is facilitated by the transport adaptor Syo1, which couples nuclear import of UL5 and UL18 with 5S rRNA association $[90,91]$. The subsequent incorporation of the 5S RNP module involves the Rpf2-Rrs1 heterodimer and has already occurred within the Nsa1-defined particle [25,86,92-94] (Figure 2K). However, the exact timing and the mechanistic details of the initial 5S RNP docking to the pre-60S ribosome remain to be determined. 
During the subsequent maturation steps, the 27SB pre-rRNA is processed within the ITS2 region: consecutive endo- and exonucleolytic cleavages catalyzed by the Las 1 complex result in the formation of the 7S and 25S' pre-rRNAs [95] (Figure 2L). Interestingly, cryo-EM structures of pre-60S ribosomes (e.g., the Nog2 particle) show in great detail how several biogenesis factors are associated with the 7S pre-rRNA to form the foot structure [25,96-98] (Figures 2M and 4). It has been proposed that these ITS2-associated factors protect the pre-rRNA and coordinate the endo- and exonucleolytic processing events [25]. Notably, these pre-60S ribosomes exhibit further structural hallmarks. The 5 S RNP is rotated by roughly $180^{\circ} \mathrm{com}-$ pared with its mature orientation [25,97]. In addition, the nascent peptidyl transferase center (PTC) is covered with biogenesis factors, including the GTPases Nog1 and Nog2, whereas the placeholder Mrt4 occupies the site of P-stalk assembly (Figures 4B and 5 ) (see below).

While navigating towards the nucleoplasm, the stepwise exonucleolytic $3^{\prime}$-end maturation of the 7S pre-rRNA is initiated by the Nop53-mediated recruitment of the exosome-assisting RNA helicase Mtr4 to the foot structure [66]. This phase is also marked by the association of the Rix1 subcomplex and the AAA-ATPase Rea1 [21,33,99] (Figures 2N and 5). Interestingly, the interaction between Rix1 and Rea1 may be regulated, as indicated by a recent study with the orthologous human proteins showing that their association is controlled by a dynamic SUMO conjugation/deconjugation cycle [100]. The cryo-EM structure of the Rea1-containing Rix1 pre-60S particle, compared with the upstream Nog2-defined pre-60S particle [25,97], revealed several major differences [33], among them the absence of the foot structure. Most notably, recruitment of the gigantic Rix1-Rea1 machinery coincides with the release of Rpf2Rrs1 and rotation of the 5S RNP into its mature position [33] (Figure 5). Once properly positioned the ATPase activity of Rea1 powers the removal of Rsa4 [21], an event that occurs

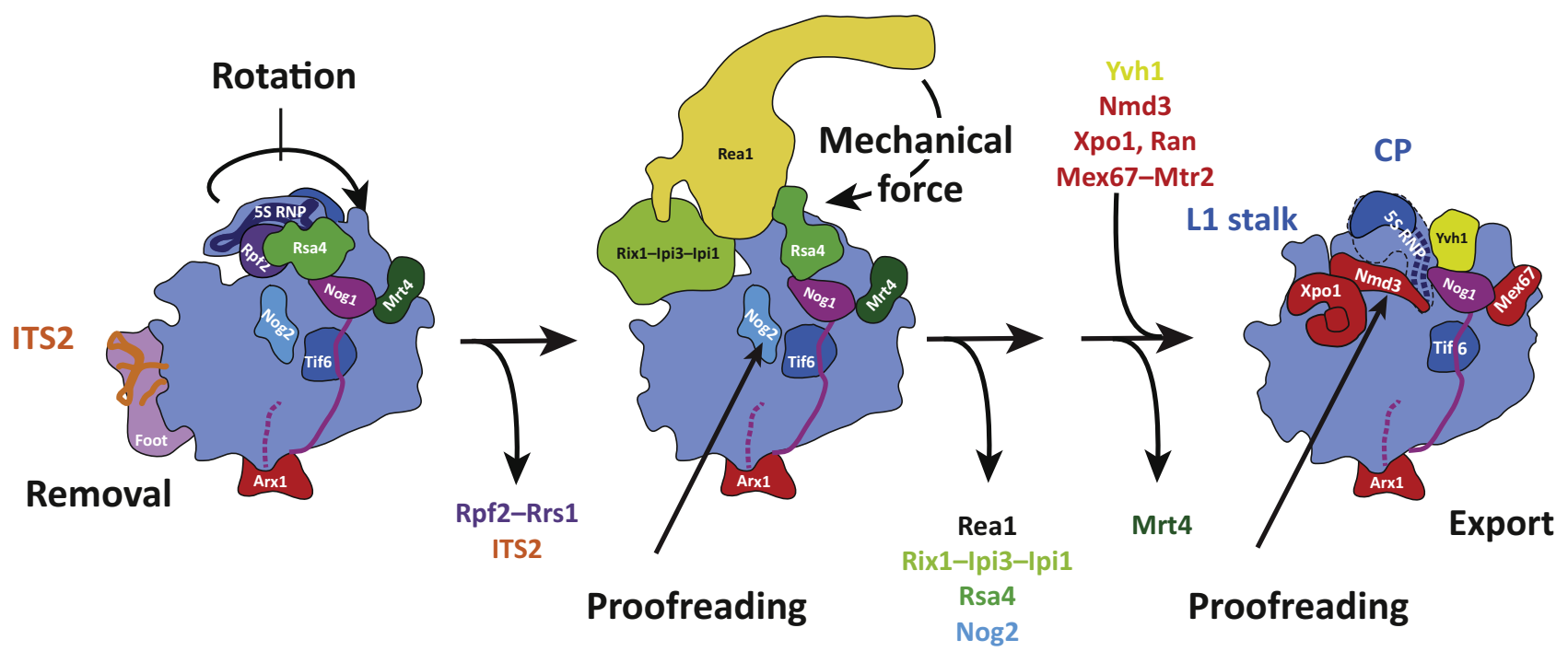

Trends in Biochemical Sciences

Figure 5. Model of Nuclear Pre-60S Maturation Events. Simplified representation of successive pre-60S intermediates that have been structurally investigated. The nucle(ol)ar Nog2-purified pre-60S particle contains the characteristic 'foot' structure [internal transcribed spacer 2 (ITS2) in orange] and the 5S ribonucleoprotein particle (RNP) is in its pre-mature conformation (left). During the next maturation steps, the exonucleolytic degradation of the ITS2 is coupled with the removal of the foot structure. Recruitment of the Rix1-Ipi3-Ipi1 subcomplex and the AAA-ATPase Rea1 induces the rotation of the 5S RNP, accompanied by the release of the Rpf2-Rrs1 heterodimer. It is proposed that Rea1 and the Rix1 subcomplex sense the correct 5S RNP position, thereby allowing the activation of Rea1's ATPase activity (middle). The resulting mechanical force towards the Rsa4-Nsa2 heterodimer induces a rRNA rearrangement at the peptidyl transferase center (PTC). Intimately coupled with this process is the activation and release of the GTPase Nog2, which appears to monitor the correct conformation around the PTC and thus provides an additional proofreading step. Release of Nog2 is a prerequisite for the recruitment of the nuclear export signal (NES)-containing export adaptor Nmd3, which is recognized by the exportin Crm1/Xpo1 in a RanGTP-dependent manner (right). The recruitment of the export factor Mex67-Mtr2 is coupled with maturation of the P stalk. Mrt4, the placeholder for $\mathrm{uL} 10$, is released by Yvh1, which allows Mex67-Mtr2 binding in proximity to the nascent $\mathrm{P}$ stalk. 
in concert with the activation and release of the GTPase Nog2 [101]. These reactions are involved in arranging the rRNA elements forming the PTC and allow recruitment of the NEScontaining export adaptor Nmd3 [30,101,102] (Figures 4B and 5). Accordingly, Rea1 and Nog2 can be viewed as checkpoint factors that sense the correct assembly status of pre-60S ribosomes; their subsequent activation then triggers remodeling events that irreversibly license these particles for export to the cytoplasm (Figures $2 \mathrm{O}$ and 5). A further checkpoint monitors the peptide exit tunnel, which is occupied by the C-terminal extension of the GTPase Nog1 in nuclear pre-60S particles [25]. However, the functional relevance of this intriguing observation needs to be tested in future studies.

\section{Nuclear Export and Cytoplasmic Maturation of Pre-60S Ribosomes}

Several export-mediating factors interact with different surfaces of pre-60S ribosomes to mediate their efficient translocation across the NPC [5,10,14,103] (Figures 20 and 5). Following the release of Nog2, the export adaptor Nmd3 binds in the A-, P-, and E-tRNA-binding sites of the LSU and recruits the exportin Crm1/Xpo1 via its essential NES sequences $[10,14,30,32,101]$. Thus, Nmd3 appears to sense the correct conformation around the PTC and prevents premature tRNA binding [30,32]. In addition, Nmd3 brings together some peripheral elements (L1 stalk, rRNA helix H38), thereby compacting the preribosome and possibly facilitating the translocation process [30]. The Mex67-Mtr2 heterodimer, another important export factor, binds pre-60S ribosomes at two distinct sites: in the neighborhood of the nascent $P$ stalk and within the $3^{\prime}$ region of the $5.8 S$ rRNA $[104,105]$. A recent study has demonstrated that recruitment and release of Mex67-Mtr2 is also intimately coupled to maturation of the emerging P stalk [104]. Besides these, several additional factors contribute to the export process $[5,10,14,103]$, most likely by shielding the charged ribosomal surface against the hydrophobic environment within the NPC channel.

After export, removal of the remaining biogenesis factors is coordinated with the incorporation of the missing r-proteins $[5,6,14,30,103]$ (Figure 2P). First, the AAA-ATPase Drg1 releases the GTPase Nog1 and its binding partner RIp24, thereby enabling recruitment of the r-protein eL24 $[99,106]$. Subsequently, Arx1 is dissociated by Jjj1 and Rei1 [103]. Interestingly, as shown by high-resolution cryo-EM, Rei1 and the closely related Reh1 probe the exit tunnel in a manner similar to Nog1 $[25,28,30]$. During P-stalk assembly, recruitment of uL10 is coupled with the release of Yvh1, which has previously displaced Mrt4 from pre-60S particles [103,104]. Release of the export adaptor Nmd3 is promoted by the GTPase Lsg1 and connected to the stable incorporation of uL16 [40,103,107] (Figure 2Q). The last biogenesis factor to be dissociated appears to be Tif6 [26,30,103,108]. Removal of this antiassociation factor is triggered by the GTPase Efl1 and is a prerequisite for subunit joining $[26,108]$. Interestingly, Efl1 and its cofactor Sdo1 largely cover the PTC region [26]; they are therefore strategically positioned to ensure that previous biogenesis events had been successfully executed. However, some of the abovementioned structures have been determined with in vitro reconstituted particles derived from mature 60S ribosomes; thus, important information about the interdependence of the cytoplasmic events might have been overlooked.

Taking these findings together, a unique feature of 605 biogenesis is the incorporation of a preassembled 5S RNP. Structural and functional investigations have already revealed fascinating insights into rRNA processing and conformational rearrangements. Finally, nuclear export and cytoplasmic maturation events are coupled with quality control checkpoints that monitor the functional integrity.

\section{Concluding Remarks and Future Perspectives}

Recent progress in the structural understanding of preribosomal particles has greatly fueled our knowledge about ribosome biogenesis. However, our structural view remains far from

\section{Outstanding Questions}

The mechanistic aspects of the early biogenesis events remain poorly understood. How are the earliest preribosomal particles formed? What are the molecular mechanisms that promote the transition from the 905 preribosome to the first pre-40S intermediate? Does the first distinct pre-60S particle form an rRNA-folding cage in a manner similar to the biogenesis-factor ensemble of the 90S preribosome? When and how is the preassembled 5S RNP incorporated into nascent pre-60S particles?

Will it be possible to obtain a structural view of additional preribosomal particles? Of special interest are high-resolution structures of early pre-60S, nuclear and cytoplasmic pre-40S, and 80S-like intermediates. These are expected to shed light on ITS1 and ITS2 processing, the transition from $90 \mathrm{~S}$ to pre-40S particles, and $40 S$ quality control prior to 205 processing

Several energy-consuming biogenesis factors are essential for ribosome assembly; however, many of these are involved in checkpoint regulations rather than in powering energetically unfavorable reactions. Is the driving force for ribosome assembly contained in the rRNA itself? Does correct folding of the rRNA create a lowerenergy state that is guided, monitored, and regulated by the associated biogenesis factors?

Ribosome assembly in prokaryotes involves only few biogenesis factors and can be reconstituted in vitro. Why is the assembly process in eukaryotes much more complicated than in prokaryotes? Will it be possible to recapitulate eukaryotic ribosome biogenesis in vitro?

Ribosomes are essential for cell growth and division. Is ribosome biogenesis an appropriate target to restrict the deregulated growth of cancer cells? Can future studies yield additional small-drug inhibitors that may have the potential to be used in medical applications? 


\section{From biochemical to structural snapshots}

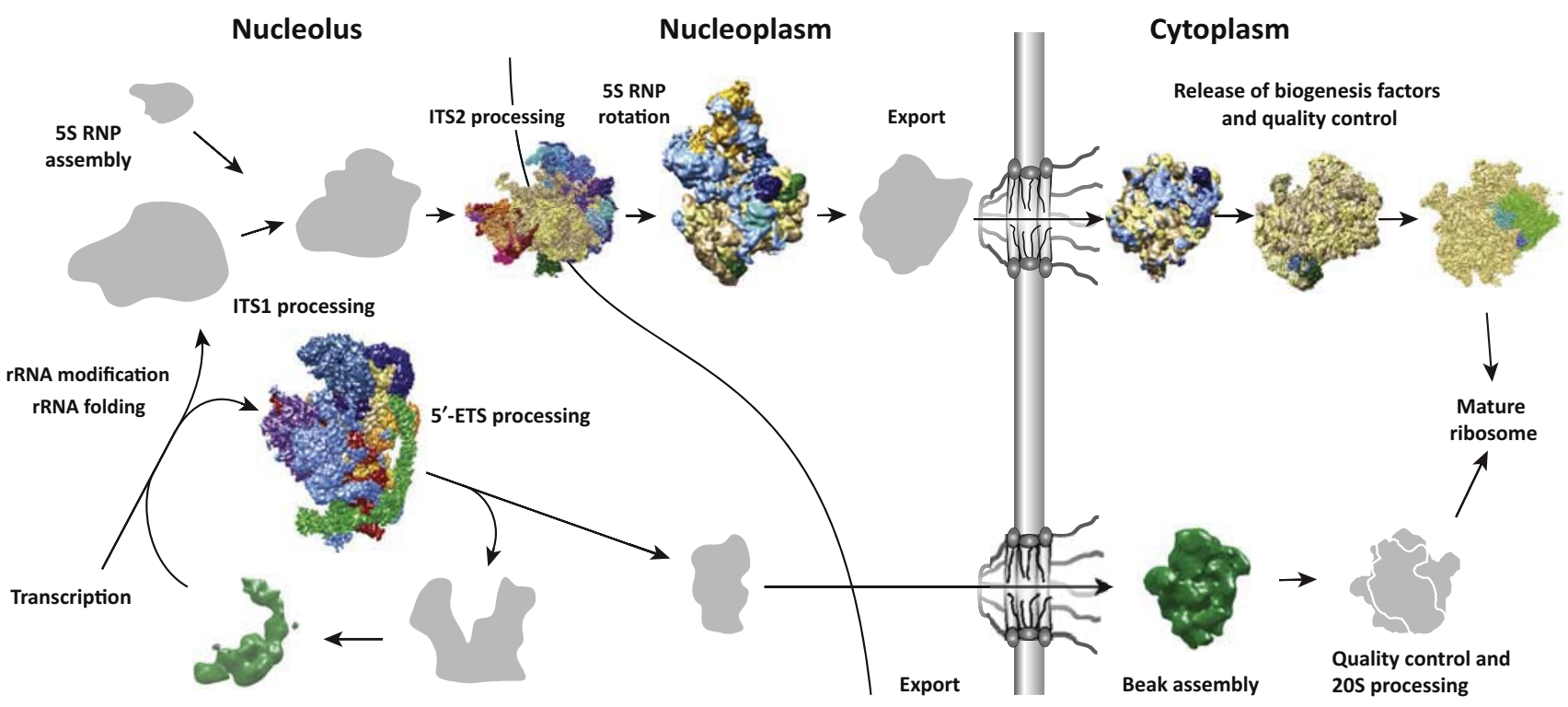

Trends in Biochemical Sciences

Figure 6. Towards a Structural View of Ribosome Assembly. Recent progress in cryoelectron microscopy (cryo-EM) has provided us with several highresolution structures of preribosomal particles. The structures of the 90S preribosome (EMD 8143, PDB 5jpq; EMD 8473, PDB 5tzs; and EMD 6695, PDB 5WYJ), the Nog2 pre-60S particle (EMD 6615, PDB 3jct), the Rea1-containing Rix1 pre-60S particle (EMD 3199, PDB 5fl8), the Yvh1 pre-60S particle (EMD 4096), and the in vitroreconstituted 60S-Arx1-Alb1-Rei1 (EMD 3151, PDB 5apo) and 60S-elF6-SBDS-EFL1 (EMD 3146, PDB 5anb) particles are shown in the context of the ribosome assembly pathway (Figure 2). For simplicity, the cryo-EM structures of the cytoplasmic, Nmd3-containing pre-60S ribosome (EMD 9569, pdb 5h4p and EMD 8362, PDB 5T62) have been omitted. Moreover, lower-resolution structures of the UTP-B module (EMD 8223) and pre-40S particles (EMD 1927; see also EMD 8346) are shown. Evidently, the structures of many important assembly modules and preribosomal intermediates remain to be determined (shown in gray).

complete and many preribosomal particles await structural determination by cryo-EM, such as the first distinct pre-40S and pre-60S intermediates (Figure 6; see Outstanding Questions). It would also be interesting to visualize 80S-like ribosomes as they subject SSUs to final quality control before their engagement in translation. We expect that future structural, enzymatic, and reconstitution approaches will continue to benefit from the utilization of thermophilic proteins and preribosomal particles from the eukaryotic organism $C$. thermophilum $[27,59,109]$. Further mechanistic and structural insights into ribosome biogenesis may promote the identification and design of small-molecule inhibitors like diazaborine or the ribozinoindoles, which act as specific inhibitors of the AAA-ATPases Drg1 and Rea1, respectively $[110,111]$. Similar to inhibitors of RNA polymerase I, such molecules are expected to have great potential for the development of novel strategies in cancer treatment [112]. In addition, solving the puzzle of ribosome assembly, by the combination of functional and structural approaches, will be instrumental for future medical advances, including our understanding of ribosomopathies.

\section{Acknowledgments}

The authors are grateful to Ingo Amm, Lisa Fromm, and Max Eivaskhani for helpful discussions and comments on the manuscript. This work was supported by the Swiss National Science Foundation (grant 31003A_156764 to D.K.) and the German Research Council (grants HU363/15-1 and HU363/12-1 to E.H. and BA2316/2-1 to J.B.). They apologize to those authors whose work could not be discussed or cited due to space limitations.

\section{References}

1. Melnikov, S. et al. (2012) One core, two shells: bacterial and 3. Ban, N. et al. (2014) A new system for naming ribosomal eukaryotic ribosomes. Nat. Struct. Mol. Biol. 19, 560-567

2. Jenner, L. et al. (2012) Crystal structure of the 80 S yeast ribo- 4. Klinge, S. et al. (2012) Atomic structures of the eukaryotic some. Curr. Opin. Struct. Biol. 22, 759-767

\author{
proteins. Curr. Opin Struct. Biol. 24, 165-169
}

ribosome. Trends Biochem. Sci. 37, 189-198 
5. Woolford, J.L., Jr and Baserga, S.J. (2013) Ribosome biogenesis in the yeast Saccharomyces cerevisiae. Genetics 195 643-681

6. de la Cruz, J. et al. (2015) Functions of ribosomal proteins in assembly of eukaryotic ribosomes in vivo. Annu. Rev. Biochem. 84, 93-129

7. Fernandez-Pevida, A. et al. (2015) Processing of preribosoma RNA in Saccharomyces cerevisiae. Wiley Interdiscip. Rev. RNA 6, 191-209

8. Turowski, T.W. and Tollervey, D. (2015) Cotranscriptional events in eukaryotic ribosome synthesis. Wiley Interdiscip. Rev. RNA 6 , 129-139

9. Thomson, E. et al. (2013) Eukaryotic ribosome biogenesis at a glance. J. Cell Sci. 126, 4815-4821

10. Nerurkar, P. et al. (2015) Eukaryotic ribosome assembly and nuclear export. Int. Rev. Cell Mol. Biol. 319, 107-140

11. Konikkat, S. and Woolford, J.L., Jr (2017) Principles of 60S ribosomal subunit assembly emerging from recent studies in yeast. Biochem. J. 474, 195-214

12. Karbstein, K. (2013) Quality control mechanisms during ribosome maturation. Trends Cell Biol. 23, 242-250

13. Panse, V.G. and Johnson, A.W. (2010) Maturation of eukaryotic ribosomes: acquisition of functionality. Trends Biochem. Sci. 35 260-266

14. Kressler, D. et al. (2010) Driving ribosome assembly. Biochim Biophys. Acta 1803, 673-683

15. Strunk, B.S. and Karbstein, K. (2009) Powering through ribosome assembly. RNA 15, 2083-2104

16. Venema, J. and Tollervey, D. (1999) Ribosome synthesis in Saccharomyces cerevisiae. Annu. Rev. Genet. 33, 261-311

17. Kressler, D. et al. (1999) Protein trans-acting factors involved in ribosome biogenesis in Saccharomyces cerevisiae. Mol. Cell. Biol. 19, 7897-7912

18. Johnson, A.W. et al. (2002) Nuclear export of ribosomal subunits. Trends Biochem. Sci. 27, 580-585

19. Tschochner, H. and Hurt, E. (2003) Pre-ribosomes on the road from the nucleolus to the cytoplasm. Trends Cell Biol. 13 255-263

20. Fatica, A. and Tollervey, D. (2002) Making ribosomes. Curr. Opin. Cell Biol. 14, 313-318

21. Ulbrich, C. et al. (2009) Mechanochemical removal of ribosom biogenesis factors from nascent $60 \mathrm{~S}$ ribosomal subunit. Cell 138, 911-922

22. Schäfer, T. et al. (2006) Hrr25-dependent phosphorylation state regulates organization of the pre-40S subunit. Nature 441, 651-655

23. Strunk, B. et al. (2011) Ribosome assembly factors preven premature translation initiation by $40 \mathrm{~S}$ assembly intermediates. Science 333, 1449-1502

24. Sengupta, J. et al. (2010) Characterization of the nuclear export adaptor protein $\mathrm{Nmd} 3$ in association with the $60 \mathrm{~S}$ ribosoma subunit. J. Cell Biol. 189, 1079-1086

25. Wu, S. et al. (2016) Diverse roles of assembly factors revealed by structures of late nuclear pre-60S ribosomes. Nature 534, $133-$ 137

26. Weis, F. et al. (2015) Mechanism of elF6 release from the nascent 60S ribosomal subunit. Nat. Struct. Mol. Biol. 22, 914-919

27. Kornprobst, M. et al. (2016) Architecture of the 90S pre-ribosome: a structural view on the birth of the eukaryotic ribosome. Cell 166, 380-393

28. Greber, B.J. et al. (2016) Insertion of the biogenesis factor Rei1 probes the ribosomal tunnel during 605 maturation. Cell 164 91-102

29. Chaker-Margot, M. et al. (2017) Architecture of the yeast smal subunit processome. Science 355, eaal1880

30. Ma, C. et al. (2017) Structural snapshot of cytoplasmic pre-60S ribosomal particles bound by Nmd3, Lsg1, Tif6 and Reh1. Nat. Struct. Mol. Biol. 24, 214-220

31. Sun, Q. et al. (2017) Molecular architecture of the $90 \mathrm{~S}$ small subunit pre-ribosome. Elife 6, e22086
32. Malyutin, A.G. et al. (2017) Nmd3 is a structural mimic of elF5A and activates the cpGTPase Lsg1 during 60S ribosome biogenesis. EMBO J. 36, 854-868

33. Barrio-Garcia, C. et al. (2016) Architecture of the Rix1?Rea1 checkpoint machinery during pre-60S-ribosome remodeling. Nat. Struct. Mol Biol. 23, 37-44

34. Lempiainen, H. and Shore, D. (2009) Growth control and ribosome biogenesis. Curr. Opin. Cell Biol. 21, 855-863

35. Albert, B. et al. (2016) A molecular titration system coordinates ribosomal protein gene transcription with ribosomal RNA synthesis. Mol. Cell 64, 720-733

36. Bange, G. et al. (2013) New twist to nuclear import: when two travel together. Commun. Integr. Biol. 6, e24792

37. Pillet, B. et al. (2017) Hold on to your friends: dedicated chaperones of ribosomal proteins: dedicated chaperones mediate the safe transfer of ribosomal proteins to their site of pre-ribosome incorporation. Bioessays 39, 1-12

38. Gamerdinger, M. (2016) Protein quality control at the ribosome: focus on RAC, NAC and RQC. Essays Biochem. 60, 203-212

39. Ting, Y.H. et al. (2017) Bcp1 is the nuclear chaperone of Rpl23 in Saccharomyces cerevisiae. J. Biol. Chem. 292, 585-596

40. Pausch, P. et al. (2015) Co-translational capturing of nascent ribosomal proteins by their dedicated chaperones. Nat. Commun. 6, 7494

41. Pillet, B. et al. (2015) The dedicated chaperone Acl4 escorts ribosomal protein Rpl4 to its nuclear pre-60S assembly site. PLoS Genet. 11, e1005565

42. Sung, M.K. et al. (2016) Ribosomal proteins produced in excess are degraded by the ubiquitin-proteasome system. Mol. Biol. Cell 27, 2642-2652

43. Sung, M.K. et al. (2016) A conserved quality-control pathway that mediates degradation of unassembled ribosomal proteins. Elife 5, e19105

44. Danilova, N. and Gazda, H.T. (2015) Ribosomopathies: how a common root can cause a tree of pathologies. Dis. Model. Mech. 8, 1013-1026

45. Farley, K.I. and Baserga, S.J. (2016) Probing the mechanisms underlying human diseases in making ribosomes. Biochem. Soc. Trans. 44, 1035-1044

46. Armistead, J. and Triggs-Raine, B. (2014) Diverse diseases from a ubiquitous process: the ribosomopathy paradox. FEBS Lett. $588,1491-1500$

47. Narla, A. and Ebert, B.L. (2010) Ribosomopathies: human disorders of ribosome dysfunction. Blood 115, 3196-3205

48. Pelava, A. et al. (2016) The importance of ribosome production, and the 5S RNP-MDM2 pathway, in health and disease. Biochem. Soc. Trans. 44, 1086-1090

49. Bursac, S. et al. (2014) Activation of the tumor suppressor p53 upon impairment of ribosome biogenesis. Biochim. Biophys. Acta $1842,817-830$

50. Watkins, N.J. and Bohnsack, M.T. (2012) The box C/D and H/ACA snoRNPs: key players in the modification, processing and the dynamic folding of ribosomal RNA. Wiley Interdiscip. Rev. RNA 3, 397-414

51. Piekna-Przybylska, D. et al. (2007) New bioinformatic tools for analysis of nucleotide modifications in eukaryotic rRNA. RNA 13, 305-312

52. Rodriguez-Galan, O. et al. (2013) Yeast and human RNA helicases involved in ribosome biogenesis: current status and perspectives. Biochim. Biophys. Acta 1829, 775-790

53. Martin, R. et al. (2013) DExD/H-box RNA helicases in ribosome biogenesis. RNA Biol. 10, 4-18

54. Phipps, K.R. et al. (2011) The small subunit processome in ribosome biogenesis - progress and prospects. Wiley Interdiscip. Rev. RNA 2, 1-21

55. Perez-Fernandez, J. et al. (2007) The $90 \mathrm{~S}$ preribosome is multimodular structure that is assembled through a hierarchical mechanism. Mol. Cell. Biol. 27, 5414-5429

56. Zhang, C. et al. (2016) Integrative structural analysis of the UTPB complex, an early assembly factor for eukaryotic small ribosomal subunits. Nucleic Acids Res. 44, 7475-7486 
57. Zhang, C. et al. (2014) Structure of Utp21 tandem WD domain provides insight into the organization of the UTPB complex involved in ribosome synthesis. PLoS One 9, e86540

58. Hunziker, M. et al. (2016) UtpA and UtpB chaperone nascent pre-ribosomal RNA and U3 snoRNA to initiate eukaryotic ribosome assembly. Nat. Commun. 7, 12090

59. Bassler, J. et al. (2017) Interaction network of the ribosome assembly machinery from a eukaryotic thermophile. Protein Sc 26, 327-342

60. Pöll, G. et al. (2014) In vitro reconstitution of yeast tUTP/UTP A and UTP B subcomplexes provides new insights into thei modular architecture. PLoS One 9, e114898

61. Zhang, L. et al. (2016) Stepwise and dynamic assembly of the earliest precursors of small ribosomal subunits in yeast. Genes Dev. 30, 718-732

62. Chaker-Margot, M. et al. (2015) Stage-specific assembly events of the 6-MDa small-subunit processome initiate eukaryotic ribosome biogenesis. Nat. Struct. Mol. Biol. 22, 920-923

63. Henras, A.K. et al. (2015) An overview of pre-ribosomal RNA processing in eukaryotes. Wiley Interdiscip. Rev. RNA 6, 225242

64. Wells, G.R. et al. (2016) The PIN domain endonuclease Utp2 cleaves pre-ribosomal RNA at two coupled sites in yeast and humans. Nucleic Acids Res. 44, 9016

65. Horn, D.M. et al. (2011) Rcl1 protein, a novel nuclease for $18 \mathrm{~S}$ ribosomal RNA production. J. Biol. Chem. 286, 34082-34087

66. Thoms, M. et al. (2015) The exosome is recruited to RNA substrates through specific adaptor proteins. Cell 162, 10291038

67. Sardana, R. et al. (2015) The DEAH-box helicase Dhr1 dissociates U 3 from the pre-rRNA to promote formation of the centra pseudoknot. PLoS Biol. 13, e1002083

68. Zhu, J. et al. (2016) Utp14 recruits and activates the RNA helicase Dhr1 to undock U3 snoRNA from the preribosome. Mol. Cell. Biol. 36, 965-978

69. Schäfer, T. et al. (2003) The path from nucleolar $90 \mathrm{~S}$ to cytoplasmic 40 S pre-ribosomes. EMBO J. 22, 1370-1380

70. Ribbeck, K. and Görlich, D. (2002) The permeability barrier of nuclear pore complexes appears to operate via hydrophobic exclusion. EMBO J. 21, 2664-2671

71. Sloan, K.E. et al. (2016) Nucleocytoplasmic transport of RNAs and RNA-protein complexes. J. Mol. Biol. 428, 2040-2059

72. Monecke, T. et al. (2014) Allosteric control of the exportin CRM unraveled by crystal structure analysis. FEBS J. 281, 4179 4194

73. Johnson, M.C. et al. (2017) Structural heterogeneity in pre-40S ribosomes. Structure 25, 329-340

74. Strunk, B.S. et al. (2012) A translation-like cycle is a quality control checkpoint for maturing 40S ribosome subunits. Cell 150, 111-121

75. Ghalei, H. et al. (2015) Hrr25/CK1 $\delta$-directed release of Ltv1 from pre-40S ribosomes is necessary for ribosome assembly and cel growth. J. Cell Biol. 208, 745-759

76. Mitterer, V. et al. (2016) Sequential domain assembly of ribosomal protein $\mathrm{S} 3$ drives $40 \mathrm{~S}$ subunit maturation. Nat. Commun. 7, 10336

77. Ferreira-Cerca, S. et al. (2012) ATPase-dependent role of the atypical kinase Rio2 on the evolving pre-40S ribosomal subunit. Nat. Struct. Mol. Biol. 19, 1316-1323

78. McCaughan, U.M. et al. (2016) Pre-40S ribosome biogenesis factor Tsr1 is an inactive structural mimic of translationa GTPases. Nat. Commun. 7, 11789

79. Ferreira-Cerca, S. et al. (2014) Dominant Rio1 kinase/ATPase catalytic mutant induces trapping of late pre-40S biogenesis factors in 80S-like ribosomes. Nucleic Acids Res. 42, 86358647

80. Turowski, T.W. et al. (2014) Rio1 mediates ATP-dependent final maturation of $40 \mathrm{~S}$ ribosomal subunits. Nucleic Acids Res. 42 12189-12199

81. Hector, R.D. et al. (2014) Snapshots of pre-rRNA structural flexibility reveal eukaryotic 40 S assembly dynamics at nucleotide resolution. Nucleic Acids Res. 42, 12138-12154
82. Lebaron, S. et al. (2012) Proofreading of pre-40S ribosome maturation by a translation initiation factor and $60 \mathrm{~S}$ subunits. Nat. Struct. Mol. Biol. 19, 744-753

83. Garcia-Gomez, J.J. et al. (2014) Final pre-40S maturation depends on the functional integrity of the 60S subunit ribosomal protein L3. PLoS Genet. 10, e1004205

84. Pertschy, B. et al. (2009) RNA helicase Prp43 and its co-factor Pfa1 promote $20 \mathrm{~S}$ to $18 \mathrm{~S}$ rRNA processing catalyzed by the endonuclease Nob1. J. Biol. Chem. 284, 35079-35091

85. Dez, C. et al. (2004) Npa1p, a component of very early pre-60s ribosomal particles, associates with a subset of small nucleolar RNPs required for peptidyl transferase center modification. Mol. Cell. Biol. 24, 6324-6337

86. Kressler, D. et al. (2008) The AAA ATPase Rix7 powers progression of ribosome biogenesis by stripping Nsa1 from pre60S particles. J. Cell Biol. 181, 935-944

87. Fatica, A. et al. (2002) Ssf1p prevents premature processing of an early pre-60S ribosomal particle. Mol. Cell 9, 341-35

88. Wehner, K.A. and Baserga, S.J. (2002) The $\sigma^{70}$-like motif: a eukaryotic RNA binding domain unique to a superfamily of proteins required for ribosome biogenesis. Mol. Cell 9, 329-339

89. Thoms, M. et al. (2016) Concerted removal of the Erb1-Ytm1 complex in ribosome biogenesis relies on an elaborate interface. Nucleic Acids Res. 44, 926-939

90. Calvino, F.R. et al. (2015) Symportin 1 chaperones 5S RNP assembly during ribosome biogenesis by occupying an essentia rRNA-binding site. Nat. Commun. 6, 6510

91. Kressler, D. et al. (2012) Synchronizing nuclear import of ribosomal proteins with ribosome assembly. Science 338, 666-671

92. Zhang. J. et al. (2007) Assembly factors Rpf2 and Rrs1 recruit $5 \mathrm{~S}$ rRNA and ribosomal proteins rpL5 and rpL11 into nascent ribosomes. Genes Dev. 21, 2580-2592

93. Madru, C. et al. (2015) Chaperoning 5S RNA assembly. Genes Dev. 29, 1432-1446

94. Kharde, S. et al. (2015) The structure of Rpf2-Rrs1 explains its role in ribosome biogenesis. Nucleic Acids Res. 43, 7083-7095

95. Gasse, L. et al. (2015) Coordinated ribosomal ITS2 RNA processing by the Las 1 complex integrating endonuclease, polynucleotide kinase, and exonuclease activities. Mol. Cell 60, 808-815

96. Wu, S. et al. (2016) Atomic modeling of the ITS2 ribosome assembly subcomplex from cryo-EM together with mass spectrometry-identified protein-protein crosslinks. Protein Sci. 26 103-112

97. Leidig, C. et al. (2014) $60 \mathrm{~S}$ ribosome biogenesis requires rotation of the $5 \mathrm{~S}$ ribonucleoprotein particle. Nat. Commun. 5, 3491

98. Bradatsch, B. et al, (2012) Structure of the pre-60S ribosoma subunit with nuclear export factor Arx1 bound at the exit tunnel. Nat. Struct. Mol. Biol. 19, 1234-1241

99. Kressler, D. et al. (2012) The power of AAA-ATPases on the road of pre-60S ribosome maturation - molecular machines that strip pre-ribosomal particles. Biochim. Biophys. Acta 1823, 92-100

100. Raman, N. et al. (2016) The AAA ATPase MDN1 acts as SUMO-targeted regulator in mammalian pre-ribosome remodeling. Mol. Cell 64, 607-615

101. Matsuo, Y. et al. (2014) Coupled GTPase and remodelling ATPase activities form a checkpoint for ribosome export. Nature 505, 112-116

102. Bassler, J. et al. (2014) A network of assembly factors is involved in remodeling rRNA elements during preribosome maturation. J. Cell Biol. 207, 481-498

103. Greber, B.J. (2016) Mechanistic insight into eukaryotic 60 S ribosomal subunit biogenesis by cryo-electron microscopy. RNA 22, 1643-1662

104. Sarkar, A. et al. (2016) Ribosome-stalk biogenesis is coupled with recruitment of nuclear-export factor to the nascent 60S subunit. Nat. Struct. Mol. Biol. 23, 1074-1082

05. Tuck, A.C. and Tollervey, D. (2013) A transcriptome-wide atlas of RNP composition reveals diverse classes of mRNAs and IncRNAs. Cell 154, 996-1009

06. Kappel, L. et al. (2012) Rlp24 activates the AAA-ATPase Drg1 to initiate cytoplasmic pre-60S maturation. J. Cell Biol. 199, $771-782$ 
107. Hedges, J. et al. (2005) Release of the export adapter, Nmd3p, from the $60 S$ ribosomal subunit requires Rpl10p and the cytoplasmic GTPase Lsg1p. EMBO J. 24, 567-579

108. Ma, C. et al. (2016) Structural dynamics of the yeast Shwachman-Diamond syndrome protein (Sdo1) on the ribosome and its implication in the 605 subunit maturation. Protein Cell 7, 187-200

109. Kellner, N. et al. (2016) Developing genetic tools to exploit Chaetomium thermophilum for biochemical analyses of eukaryotic macromolecular assemblies. Sci. Rep. 6, 20937
110. Kawashima, S.A. et al. (2016) Potent, reversible, and specific chemical inhibitors of eukaryotic ribosome biogenesis. Cell 167 , 512-524 e514

111. Loibl, M. et al. (2014) The drug diazaborine blocks ribosome biogenesis by inhibiting the AAA-ATPase Drg1. J. Biol. Chem. 289, 3913-3922

112. Quin, J.E. et al. (2014) Targeting the nucleolus for cancer inter vention. Biochim. Biophys. Acta 1842, 802-816

113. Ben-Shem, A. et al. (2011) The structure of the eukaryotic ribosome at $3.0 \AA$ A resolution. Science 334, 1524-1529 